\title{
Prospects for a novel ultrashort pulsed laser technology for pathogen inactivation
}

\author{
Shaw-Wei D Tsen" ${ }^{1}$, Tzyy Choou Wu ${ }^{2,3,4,5}$, Juliann G Kiang ${ }^{6,7,8}$ and Kong-Thon Tsen ${ }^{\text {* }^{*}}$
}

\begin{abstract}
The threat of emerging pathogens and microbial drug resistance has spurred tremendous efforts to develop new and more effective antimicrobial strategies. Recently, a novel ultrashort pulsed (USP) laser technology has been developed that enables efficient and chemical-free inactivation of a wide spectrum of viral and bacterial pathogens. Such a technology circumvents the need to introduce potentially toxic chemicals and could permit safe and environmentally friendly pathogen reduction, with a multitude of possible applications including the sterilization of pharmaceuticals and blood products, and the generation of attenuated or inactivated vaccines.
\end{abstract}

\section{Review}

Despite the myriad antimicrobial methods that have been developed to combat infectious disease, microbial pathogens continue to evolve and acquire resistance. In addition, emerging pathogens such as Human Immunodeficiency Virus (HIV) [1] in the 1980s and more recently West Nile Virus (WNV) [2] continue to pose threats before testing and containment strategies are in place. Therefore, new and more effective pathogen inactivation strategies are urgently needed.

Use of Ultrashort pulsed (USP) lasers for selective disinfection has emerged as a potentially attractive antimicrobial strategy. USP laser treatment has been shown to inactivate a variety of viruses including HIV, Influenza virus, Human Papillomavirus (HPV), Murine Noroviruses, Hepatitis A Virus (HAV), Encephalomyocarditis Virus (EMCV), Tobacco Mosaic Virus (TMV) and M13 bacteriophage, as well as bacteria such as E. coli, Salmonella spp, and Listeria [3-11].

The USP laser technology has the following advantages over the current methods of disinfection of pathogens:

(1)With conventional pharmaceutical antiviral and antibacterial treatments, a new drug is usually required to combat new or mutated strains of microorganisms. In contrast, the USP laser method is effective for the inactivation of enveloped and non-enveloped, single-stranded, double-stranded

\footnotetext{
* Correspondence: tsen@asu.edu

${ }^{9}$ Department of Physics, Arizona State University, Tempe, AZ 85287, USA

Full list of author information is available at the end of the article
}

DNA, RNA viruses, and gram-positive and gramnegative bacteria [3-11], suggesting that the USP laser technique could represent a general method for inactivating viral and bacterial pathogens regardless of their structural composition or mutation status. For the inactivation of a virus, the USP laser method excites mechanical vibrations of the capsid of a virus and targets the weak links of the viral protein coat, leading to its loss of infectivity; for the inactivation of a bacterium, the USP laser technique relaxes the super-coiled doublestranded DNA causing damage and subsequent death of the bacterium. This is demonstrated by the results in Table 1 [3-11] in which a variety of viruses and bacteria have been shown to be efficiently inactivated by the USP lasers.

(2) Existing disinfection methods such as irradiation of ultraviolet (UV) light, gamma-ray, UV/photochemicals, microwave absorption, and pharmaceutical antiviral and antibacterial treatments are not selective; as a result, severe side effects may accompany the treatments. On the other hand, the USP laser method has been shown $[3,6,9]$ to inactivate undesired microorganisms like viruses and bacteria while leaving desired materials such as mammalian cells and proteins unharmed; i.e., the USP laser technique is capable of selective disinfection and therefore has minimal potential side effects. Table 2 shows experimental results on the selectivity of a near-infrared USP laser on a variety of microorganisms. The intriguing feature worthwhile mentioning is that there exists a

\section{() Biomed Central}


Table 1 Killing efficacy for a variety of microorganisms using A $425 \mathrm{~nm}$ - femtosecond pulsed laser (laser exposure time = 3.6 seconds)

\begin{tabular}{lll}
\hline Microorganism & Properties & Load reduction \\
\hline Human Immunodeficiency Virus (HIV) & Enveloped, single-stranded RNA & $10^{4}$ \\
\hline Influenza Virus & Enveloped, single-stranded RNA & $10^{5}$ \\
\hline Encephalomyocarditis virus (EMCV) & Non-enveloped, single-stranded RNA & $10^{3}$ \\
\hline Murine norovirus (MNV) & Non-enveloped, single-stranded RNA & $10^{3}$ \\
\hline Hepatitis A virus (HAV) & Non-enveloped, single-stranded RNA & $10^{3}$ \\
\hline Human Papillomavirus (HPV) & Non-enveloped, double-stranded DNA & $10^{5}$ \\
\hline M13 bacteriophage & Non-enveloped, single-stranded DNA & $10^{5}$ \\
\hline Escherichia coli & Gram negative & $10^{4}$ \\
\hline Salmonella typhi & Gram negative & $10^{5}$ \\
\hline Listeria monocytogenes & Gram positive & $10^{3}$ \\
\hline Enterobacter Sakazakii & Gram negative & $10^{3}$ \\
\hline
\end{tabular}

therapeutic window in laser power density between 1 $\mathrm{GW} / \mathrm{cm}^{2}$ and $10 \mathrm{GW} / \mathrm{cm}^{2}$ which allows the inactivation of a variety of pathogens while leaving mammalian cells unharmed. The existence of this window enables selective inactivation of microorganisms.

(3) Because of the nature of USP laser inactivation, the USP laser technique is sensitive to the global oscillation of the capsid but not to minor changes caused by nucleic acid mutation in the pathogens; as a result the USP laser technology can be used to inactivate both wildtype and mutated/drug-resistant strains of microorganisms. An example is given for M13 bacteriophages in which both wild-type and engineered strains are efficiently inactivated by the irradiation of USP lasers [9]. This intriguing feature makes the USP laser technique particularly suitable for the disinfection of rapidly evolving or drug-resistant viral and bacterial species such as HIV and MRSA, respectively.

(4) Currently available pathogen reduction methods for blood components usually involve the addition of potentially toxic or carcinogenic chemicals. Residual amounts of these chemicals can remain within the transfusion products and then be transfused. In addition, it is likely that in some cases these chemicals may interact with the product itself,

Table $\mathbf{2}$ Threshold laser power density for inactivation of viruses and cells

\begin{tabular}{|c|c|c|c|c|c|c|c|}
\hline & \multicolumn{7}{|c|}{ Viruses and Cells } \\
\hline & M13 & TMV & HPV & HIV & $\begin{array}{l}\text { Human } \\
\text { red } \\
\text { blood } \\
\text { cell }\end{array}$ & $\begin{array}{l}\text { Human } \\
\text { Jurkat } \\
\text { T-cell }\end{array}$ & $\begin{array}{l}\text { Mouse } \\
\text { dendritic } \\
\text { cell }\end{array}$ \\
\hline $\begin{array}{l}\text { Threshold } \\
\text { Laser } \\
\text { Power Density } \\
\text { for inactivation } \\
\left(\mathrm{GW} / \mathrm{cm}^{2}\right)\end{array}$ & 0.06 & 0.85 & 1.0 & 1.1 & 15 & 22 & 12 \\
\hline
\end{tabular}

potentially altering its structure or function. The potential side effects due to the introduction of such chemicals during the pathogen reduction process is a major concern from the FDA standpoint [12] On the other hand, the USP laser technology is chemical-free; in other words, it does not involve introducing chemicals during pathogen reduction. This makes the USP laser method safe and environmentally friendly, and advantageous for treating products such as blood products, pharmaceuticals, therapeutics, vaccines, and other agents that are used in humans.

\section{Basic mechanism of inactivation of pathogens by ultrashort pulsed lasers}

\section{Inactivation of a virus by ultrashort pulsed lasers}

We take M13 as an example for demonstration. Figure 1 shows plaque forming units (pfu) as a function of laser power density for M13 bacteriophages excited by a near-infrared Ti-sapphire cw mode-locked laser $[4,5,7]$ The intriguing feature of these assay results is the rapid cut-off of the pfu of M13 bacteriophages at around $60 \mathrm{MW} / \mathrm{cm}^{2}$. A similar feature (which is not shown here) is also found when a visible USP laser is used for inactivation. This unique feature of inactivation upon laser power density indicates the emergence of a new virus inactivation mechanism for M13 bacteriophages by the irradiation of USP lasers - impulsive stimulated Raman scattering (ISRS) - which is elucidated below.

The atomic force microscope (AFM) images from the control and laser treated M13 bacteriophage samples provide an important clue for the inactivation mechanism. The AFM images of a M13 bacteriophage sample before and after the visible USP laser irradiation are shown in Figure 2(a) and 2(b), respectively [10]. The relatively smooth worm-like features having a diameter of about $6 \mathrm{~nm}$ and about $850 \mathrm{~nm}$ in length in Figure 2(a) 


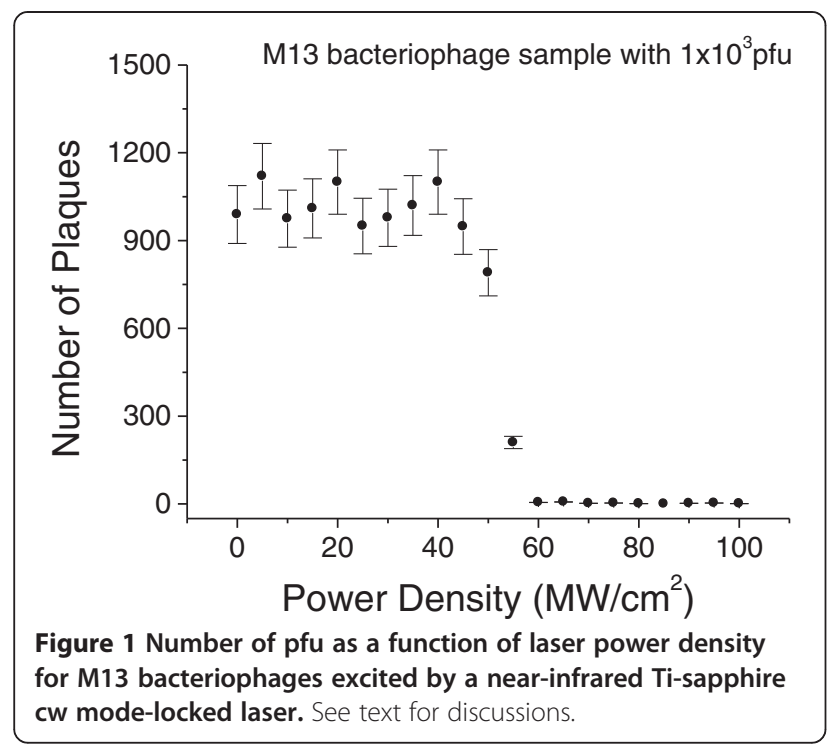

revealed the presence of M13 bacteriophages in the control. Figure (b) showed, in contrast to Figure 2(a), the appearance of many small structures which were about 6 $\mathrm{nm}$ in diameter after laser irradiation. As discussed later, these small structures were consistent with the size of individual $\alpha$-helix protein units of which the protein capsid of the M13 bacteriophage is composed. As a result, these small structures are attributed to individual $\alpha$-helix protein units of the M13 bacteriophage. In addition, some zigzagged worm-like features (encircled by artificially drawn black curves for the sake of clarity) were observed. The fact that its length was about 850 $\mathrm{nm}$ and that it was in a zigzagged structure indicated that these zigzagged structures were naked viral genomic DNAs from M13 bacteriophages. The observation of the naked DNAs in the laser-irradiated M13 bacteriophage sample indicated that irradiation of the visible USP laser severely altered the structural integrity of the protein shell of the M13 bacteriophages, potentially causing the DNA to "leak out".

By taking into account the size of small structures about $6 \mathrm{~nm}$ in diameter in the AFM images of M13 bacteriophages after USP laser irradiation in Figure 2(b), the resolution of the tip of AFM used in the imaging, and the actual size of the $\alpha$-helix protein unit which forms the capsid of a M13 bacteriophage, we have found that the small structures observed in Figure 2(b) are consistent in size with those of the $\alpha$-helix protein units of the capsid of M13 bacteriophages. This analysis further supports our conclusion that USP laser irradiation under our experimental conditions does not damage individual protein units in M13 bacteriophages.

Figure 3 shows the result from agarose gel electrophoresis on single-stranded DNAs from M13 bacteriophages (control) and from M13 bacteriophages irradiated with a visible USP laser [10]. The laser-irradiated M13 bacteriophage sample showed a single dark band similar in width to and located at the same position as that of the control sample. Therefore, these experimental results indicated that, within experimental uncertainty, irradiation of a visible USP laser caused no severe structural change of singlestranded DNAs of M13 bacteriophages. In other words, the gel electrophoresis results of Figure 3 on the single-stranded DNAs of M13 bacteriophages indicate

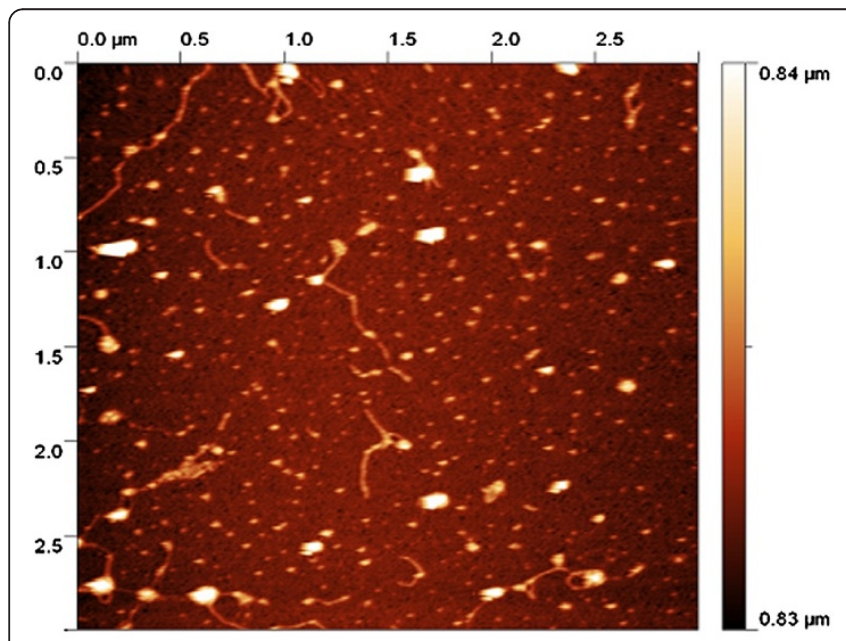

(a)

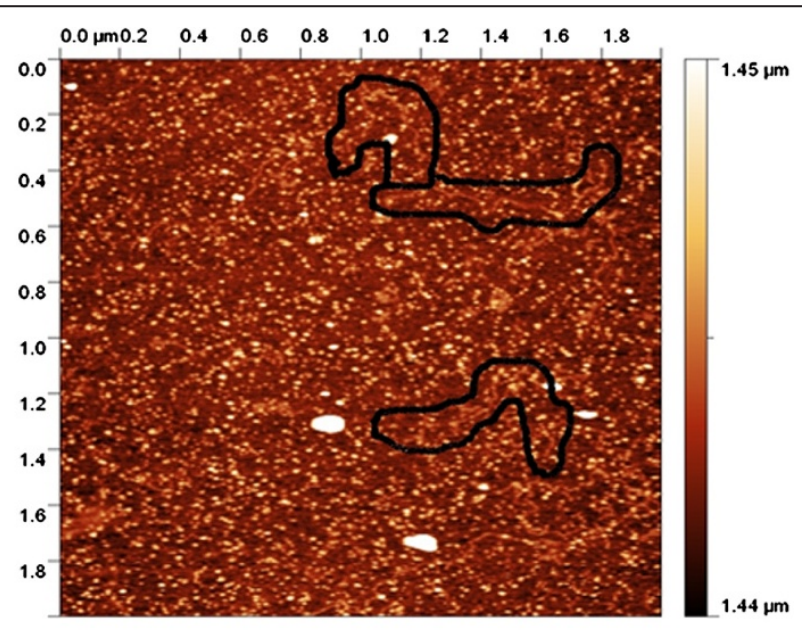

(b)

Figure 2 Atomic Force Microscope images of M13 bacterioaphages (a) without laser irradiation and (b) with laser irradiation by a visible femtosecond laser. For clarity, the black curves in (b) were drawn to encircle the bare DNAs. See text for discussions (with publisher's permission) 
that irradiation of a visible USP laser does not significantly alter the structure of single-stranded DNA.

The luminescence, excitation, and circular dichroism (CD) spectra from amino acids of proteins are very sensitive to the structural changes of proteins. Therefore, these optical characterization methods were employed to detect the primary and secondary structural changes of proteins before and after the visible USP laser irradiation. Figures 4(a), 4(b), 4(c) show our preliminary results for bovine serum albumin (BSA) proteins in buffer solution with and without irradiation with an USP laser [10]. In Figure 4(a), the excitation spectrum corresponded to the broad structure centered around 280 $\mathrm{nm}$. The luminescence spectrum represented the broad peak around $340 \mathrm{~nm}$. Each spectrum contained 4 curves in which two of them were control and two were laserirradiated samples, as indicated. The two control samples and two laser-irradiated samples had $60 \mu \mathrm{M}$, $300 \mu \mathrm{M}$ of BSA proteins, respectively. For clarity, the spectra shown were normalized to the concentration of BSA proteins. In Figure 4(b), the far UV CD contained four curves, in which two of them were control and two were laser-irradiated samples. The two control samples and two laser-irradiated samples had $60 \mu \mathrm{M}, 300 \mu \mathrm{M}$ of BSA proteins, respectively. For clarity, the spectra shown were normalized to the concentration of BSA proteins. In Figure 4(c), the near UV CD included four curves in which two of them were control and two were laser-irradiated samples. The two control samples and two laser-irradiated samples had $60 \mu \mathrm{M}, 300 \mu \mathrm{M}$ of BSA proteins, respectively.
For clarity, the spectra shown were normalized to the concentration of BSA proteins. The experimental results show that, within experimental uncertainty, the luminescence, excitation spectra and circular dichroism of BSA proteins remained practically the same before and after the laser irradiation, indicating minimal or no structural changes in BSA proteins after irradiation with a visible USP laser. Therefore, these experimental results on the optical characterization of BSA proteins suggest that there is virtually no structural change in BSA proteins upon USP laser irradiation. Because BSA is primarily made up of $\alpha$ helix proteins, and the capsid of a M13 bacteriophage is mostly composed of $\alpha$-helix protein units, these results suggest that the visible USP laser irradiation will not damage the individual protein units that comprise the protein capsid of M13 bacteriophage.

Thus, the AFM images of Figure 2 together with the DNA gel electrophoresis results of Figure 3 and optical results of BSA proteins of Figure 4 are consistent with our model: that irradiation with a USP laser alters the structural integrity of the protein capsid of M13 bacteriophages by disrupting weak interactions between proteins without damaging either the viral genomic single-stranded DNA or the individual protein units of M13 bacteriophage capsid.

Irradiation with an intense ultrashort pulsed laser such as a femtosecond laser can deposit laser energy onto the protein capsid of a viral particle by the excitation of lowfrequency acoustic vibrations on the capsid of a virus. This process, known as impulsive stimulated Raman 


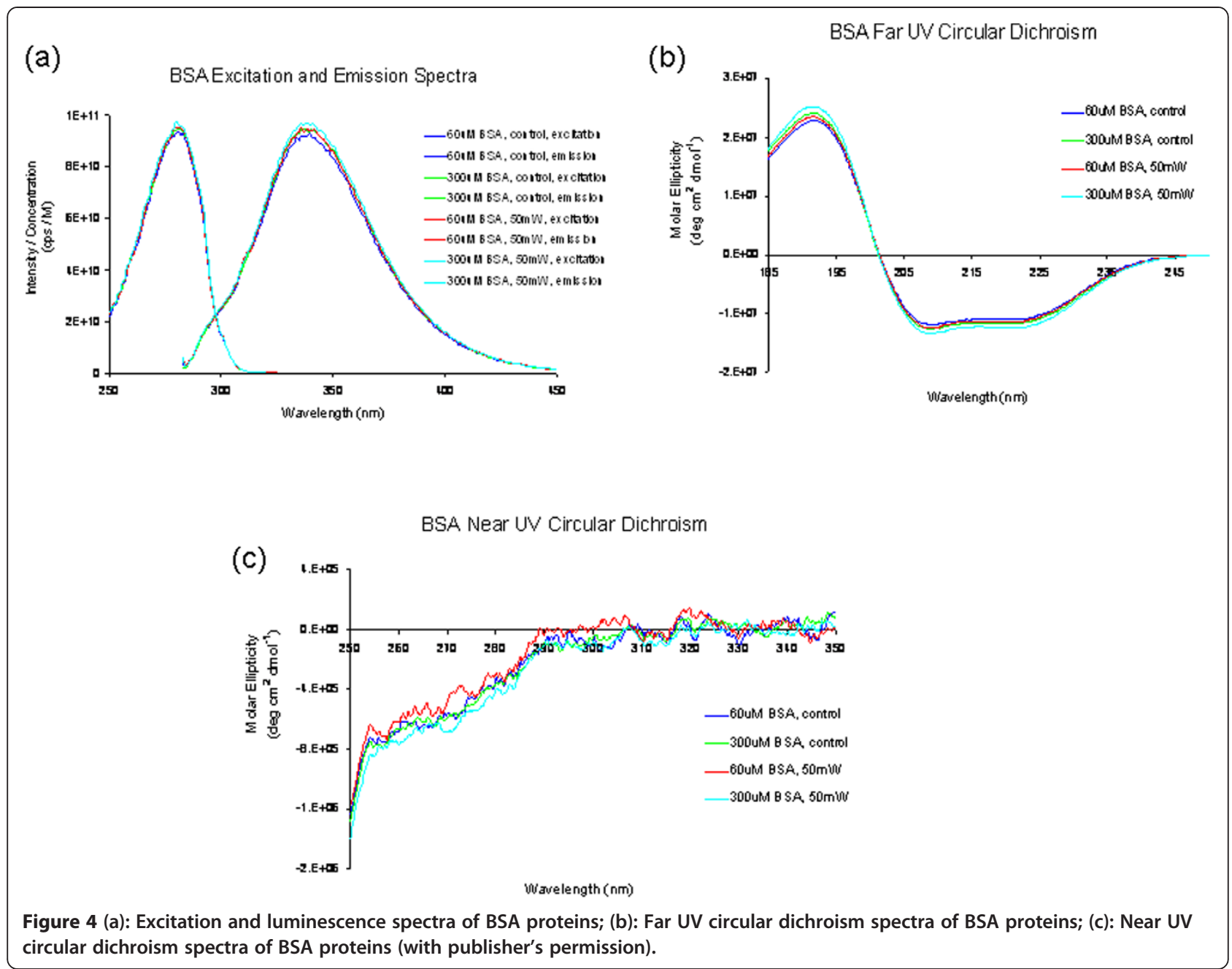

scattering (ISRS), has been used to deposit laser energy to solid state systems as well as to biological molecules [13-20].

The ISRS process can be understood as follows:

The vibrational mode of a macromolecule such as a virus excited by the laser is represented by normal coordinate $\mathrm{Q}$. If we ignore dispersion in the index of refraction and assume that the incident electric field from the excitation laser is not depleted by the stimulated scattering, the equation of motion for $\mathrm{Q}$ can be written as $[21,22]$

$$
\frac{\partial^{2} Q}{\partial t^{2}}+2 \gamma \frac{\partial Q}{\partial t}+\omega_{0}^{2} Q=f(t)
$$

where $\omega_{0}$ is the angular frequency of vibration, $\gamma$ is the damping constant and $f(t)$ is the impulsive driving force produced by the excitation laser and is described next.
The electric field $\vec{E}_{L}$ of the laser induces a polarization on the molecule due to its polarizability $\alpha$ as $\vec{P}=\alpha \vec{E}_{L}$, where for simplicity we neglect the tensor properties of $\alpha$. The polarizability has a static part that produces elastic Rayleigh scattering, and a part that is modulated by the oscillating displacement $\mathrm{Q}$. It is this modulated contribution that produces the Raman effect and the ISRS process in the macromolecule. The polarizability $\alpha$, expanded in a Taylor series in Q, is

$$
\alpha(Q)=\alpha_{0}+\alpha_{0}^{\prime} Q+\frac{1}{2} \alpha_{0}^{\prime \prime} Q^{2}+
$$

higher order terms in $\mathrm{Q}$ (2); where $\alpha 0$ is the zero order term $\alpha_{0}{ }^{\prime} Q \equiv\left(\frac{\partial \alpha}{\partial Q}\right)_{0} Q$ is the first order term resulting in the first order Raman scattering processes; $\frac{1}{2} \alpha_{0}{ }^{\prime \prime} Q^{2} \equiv \frac{1}{2}\left(\frac{\partial^{2} \alpha}{\partial Q^{2}}\right)_{0} Q^{2}$ is the second order term, etc.

The potential energy stored in an induced polarization is $U(Q, t)=-\frac{1}{2} \vec{P}(Q, t) \cdot \vec{E}_{L}(t)$. If we keep up to the first 
order term and neglect the second order and higher order terms in the polarization expansion in Eq, (2), the generalized driving force $f(t)=-\frac{\partial U(Q, t)}{\partial Q}$ on the right hand side of Eq. (1) becomes

$$
f(t)=\frac{1}{2} \alpha_{0}^{\prime} E_{L}^{2}
$$

Equation (1) with $f(t)$ given by Eq. (3) can be solved by using Green's function method to determine the normal coordinate $\mathrm{Q}(\mathrm{t})[13,23]$. In particular, for excitation by a single-beam ultrashort laser having a pulse width of $\tau_{L}$, and intensity $I(t)=I_{0} \cdot e^{-\left(t^{2} / \tau_{L}^{2}\right)}$, assuming small damping, the displacement is $Q(t)=Q_{0} e^{-\gamma t} \sin \left(\omega_{0} t\right)$. Of greatest importance in $Q(t)=Q_{0} e^{-\gamma t} \sin \left(\omega_{0} t\right)$ is the amplitude $Q_{0}$ of the displacement away from the equilibrium position of the molecule produced by ISRS process, which is given by $[13,23]$

$$
Q_{0}=\frac{\sqrt{\pi}}{2} \frac{n}{c K \epsilon_{0}} \alpha_{0}^{\prime} \frac{\tau_{L}}{\omega_{0}} \cdot I_{0} \cdot e^{-\left(\omega_{0}^{2} \tau_{L}^{2} / 4\right)} .
$$

Here $I_{0}$ is the peak intensity of the excitation laser, $\alpha_{0}^{\prime}$ is the polarizability derivative proportional to the amplitude of the Raman scattering cross section, $n$ is the index of refraction, $\mathrm{c}$ the speed of light, and $K \epsilon_{0}$ the permittivity of the dielectric medium.

Therefore, in this ISRS process, the deposited laser energy on the protein capsid of a viral particle is proportional to the square of the laser intensity and to the Raman scattering cross section. If the deposited laser energy or the amplitude of the excited resonance mode on the capsid of a viral particle is large enough, it can break the weak links (for example, hydrogen bonds or hydrophobic contacts) between the proteins, damage to the capsid of the virus occurs, leading to the viral inactivation.

In the ISRS process, operated in near-infrared/visible wavelength range to which water is transparent, one way of selective killing of microorganisms is by varying the laser power density; the other way of selective killing of microorganisms in biological systems is by controlling the range of spectral content of an ultrashort pulsed laser. For a transform-limited pulsed laser, by using Heisenberg uncertainty principle, it is equivalent to controlling the laser pulse width. The presence of the factor $e^{-\omega_{0}{ }^{2} \tau_{L}{ }^{2} / 4}$ in Eq. (4) indicates that in order to excited significantly large amplitude $Q_{0}$ of a vibrational frequency $\omega_{0}$ in a microorganism for damaging effect, the excitation laser pulse width $\tau_{L}$ has to be chosen so that $\omega_{0} \tau_{L}$ $\leq 1$. Because each microorganism has its own characteristic resonance vibrational frequency $\omega_{0}$, by choosing the proper pulse width of an ultrashort pulsed laser, the amplitude of this resonance mode can be excited so high as to damage and inactivate the microorganism.

We note that cw (continuous wave) laser cannot excite the resonance mode $\omega_{0}$ of a microorganism through an ISRS process. Because $\tau_{L}=\infty$ for a cw laser, Eq. (4) therefore indicates that the amplitude of the excited vibrational mode is zero. A Q-switched laser cannot excite the resonance mode $\omega_{0}$ of a typical microorganism through ISRS process either. This is because each microorganism has a characteristic resonance vibrational frequency $\omega_{0}$ which typically is in the range of $100 \mathrm{GHz}$; [24-29] for example, helix-shaped M13 bacteriophage is around $300 \mathrm{GHz}$ [27-29] and icosahedral viruses of 30 $\mathrm{nm}$ in size like murine norovirus is around $65 \mathrm{GHz}$ [24] and if we use a viral frequency of $100 \mathrm{GHz}$ and the fact that a typical Q-switched laser has a pulse width of about 100 nanosecond, from Eq. (4), the factor $e^{-\left(\omega_{0}{ }^{2} \tau_{L}{ }^{2} / 4\right)}$ becomes vanishingly small. Therefore, the amplitude of vibrations a Q-switched laser will excite is negligibly small.

The rapid switch from non-inactivation to inactivation at the laser power density of $60 \mathrm{MW} / \mathrm{cm}^{2}$ shown in Figure 1 for M13 bacteriophages can be explained by the ISRS process. When the laser power density is small $\left(<60 \mathrm{MW} / \mathrm{cm}^{2}\right)$, the excited amplitude of vibration on the capsid of M13 bacteriophage is not large enough to break the weak links and no inactivation is observed; however, as the laser power density increases to and beyond $60 \mathrm{MW} / \mathrm{cm}^{2}$, the excited amplitude of vibration becomes large enough to break the weak links on the capsid of the M13 bacteriophage, leading to the inactivation of M13 bacteriophage.

To further support our argument that viral particles are inactivated by the irradiation of USP lasers through an ISRS process, we show experimental results of the inactivation of M13 bacteriophages as a function of laser pulse widths/spectral widths in Table 3 [4-7] while the laser intensity is kept constant. The abrupt change from inactivation to no inactivation observed in the experiments when the pulse width of the laser changes from $500 \mathrm{fs}$ to $800 \mathrm{fs}$ is consistent with the prediction of Eq. (4) by using the Raman mode frequency of $10 \mathrm{~cm}^{-1}$ which was measured by Raman spectroscopy for M13 bacteriophages [27-29].

Therefore, schematically, this is what is happening in our model for USP laser inactivation of viruses such as

Table 3 Dependence of the status of M13 bacteriophage on laser pulse width

\begin{tabular}{llllll}
\hline Pulse Width (fs) & 80 & 250 & 500 & 800 & 1000 \\
\hline Spectral Width $\left(\mathrm{cm}^{-1}\right)$ & $(80)$ & $(25)$ & $(12)$ & $(6.5)$ & $(5)$ \\
\hline Status Inactivation (Yes or No) & Yes & Yes & Yes & No & No \\
\hline
\end{tabular}

(The excitation laser intensity is kept at $5.6 \times 10^{-6} \mathrm{~J} / \mathrm{cm}^{2}$ ).

(The numbers with in the brackets indicate the spectral width in $\mathrm{cm}^{-1}$ ). 

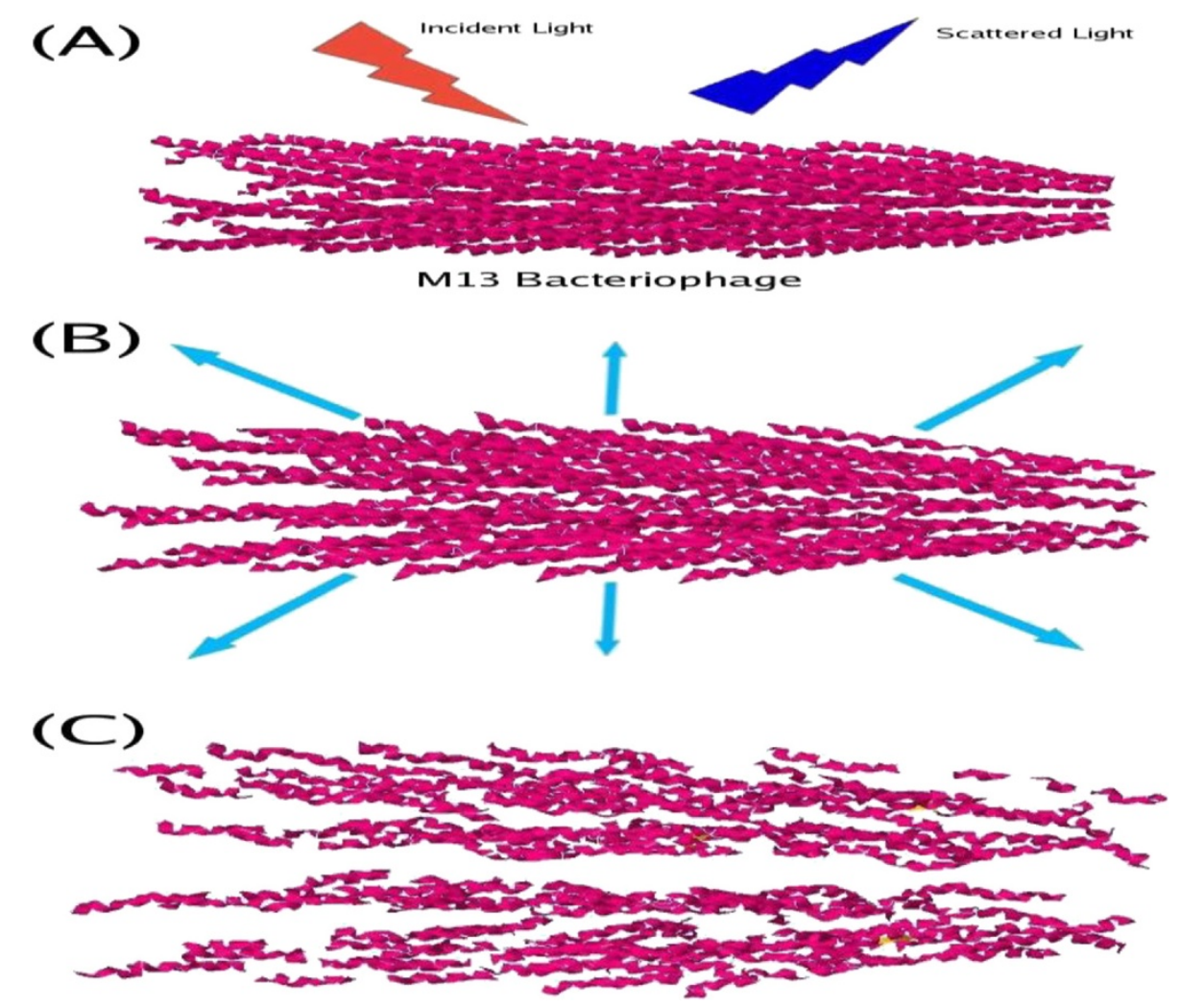

Figure 5 Diagrams showing how the M13 bacteriophage is inactivated by an USP laser. (A) The electric field from a femtosecond laser produces an impulsive force through the induced charge polarization on the virus; (B) The resultant mechanical impact coherently excites Raman-active vibrational modes on the capsid of the virus; (C) If the pulse width/spectral width and intensity of the USP laser are appropriately chosen, the vibrational modes can be excited to such high energy states as to break off the weak links between proteins in the capsid of the virus, damaging/disintegrating the capsid and leading to the inactivation of the virus.

the M13 bacteriophage: The electric field from a femtosecond laser produces an impulsive force through the induced charge polarization on the virus, as shown in Figure 5(A). This mechanical impact coherently excites Raman-active vibrational modes on the capsid of the virus, as depicted in Figure 5(B). Figure 5(C) demonstrates that if the pulse width/spectral width and intensity of the USP laser are appropriately chosen, the vibrational modes can be excited to such high energy states as to break off the weak links on the capsid of the virus, damaging/disintegrating the capsid and leading to the inactivation of the virus.

\section{Inactivation of bacteria by ultrashort pulsed lasers}

We take Salmonella typhimurium as an example. To obtain insight into the inactivation mechanisms, we have performed inactivation of a mutant Salmonella typhimurium by a visible USP laser. The mutant is deficient in RecA proteins which are responsible for the repair of damaged DNA. In other words, the mutant is very sensitive/vulnerable to the damage of DNA. Figure 6 [10] shows the inactivation of both the wild-type and mutant
Salmonella typhimurium by a visible USP laser as a function of the laser fluence. In general, the log - load reduction factor at a given laser dose has be found to be

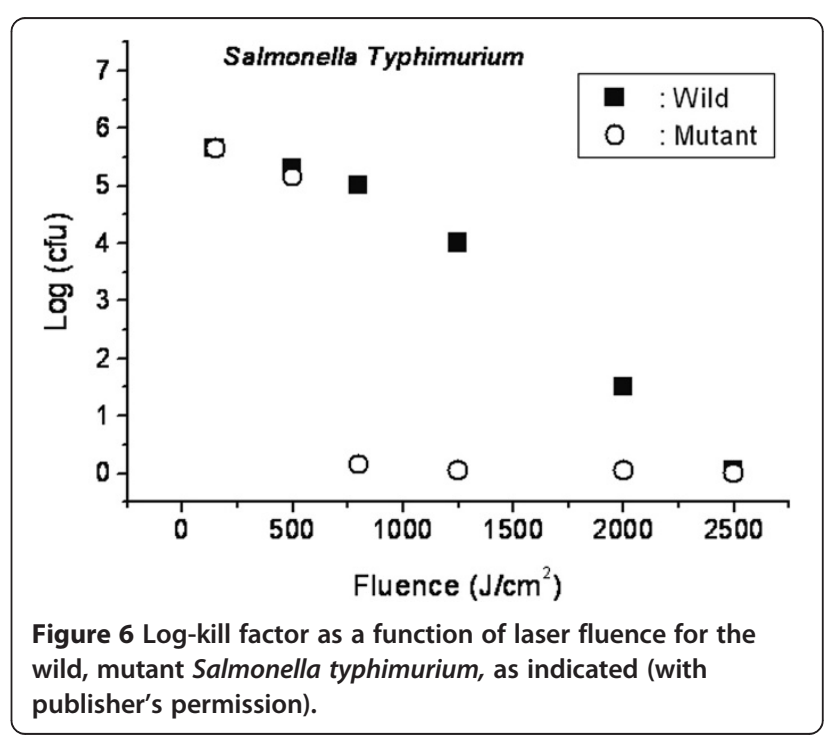


higher for the mutant than for the wild strain. In particular, our experimental results indicate that by using the USP laser, with laser dose of about $800 \mathrm{~J} / \mathrm{cm}^{2}$, a log load reduction factor of about 5 for mutant Salmonella typhimurium was observed; however, by employing the same laser parameter, a log-kill factor of only 0.5 for the wild Salmonella typhimurium was found. Because the only difference between these two strains of Salmonella typhimurium is the RecA proteins which are in charge of the repair of damaged DNA, these experimental results indicate that irradiation of a visible USP laser causes DNA damage and subsequent inactivation of the Salmonella typhimurium.

Figure 7 demonstrates our preliminary results for isolated double-stranded DNAs in buffer solution before and after irradiation by a visible femtosecond laser, as detected by the agarose gel electrophoresis method [10]. The control sample (labeled No. 1) revealed the presence of three dark bands corresponding to circular, linear, and super-coiled double-stranded DNA, respectively. Sample No. 2 showed that stirring the sample slightly changed the relative darkness of the bands. On the other hand, the laser-irradiated sample (labeled No. 3) showed that the relative darkness of the three bands was greatly

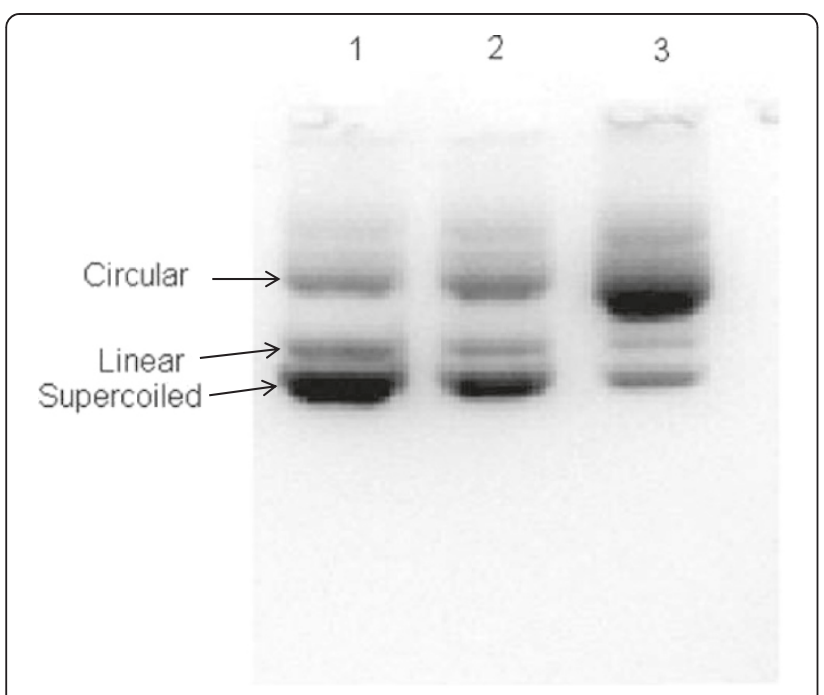

\section{Double-stranded DNA (Control) 2. After magnetic stirring \\ 3. After laser irradiation and stirring}

Figure $7 \mathrm{Gel}$ electrophoresis experiments on double-stranded DNAs. \#1 is the control without magnetic stirring showing the presence of super-coiled, linear and circular DNAs; \#2 is another control with magnetic stirring; \#3 is the laser-irradiated sample with magnetic stirring. The visible femtosecond laser is operated at 425 $\mathrm{nm}$, at a repetition rate of $80 \mathrm{MHz}$, with an average power of 100 $\mathrm{mWs}$, laser spot size of about 100 micron, and laser irradiation time of $1 \mathrm{hr}$. altered. These data suggest that the effects of visible femtosecond laser irradiation primarily caused relaxation of the supercoiled double-stranded DNA to produce relaxed circular double-stranded DNA. Because forced changes in the supercoiling status of DNA can disrupt cellular metabolism, which can lead to the death of the cell, one mechanism which can contribute to the inactivation of Salmonella typhimurium by the irradiation of a visible USP laser is relaxation of supercoiled DNA in the bacteria.

It has been known that photo-stimulation of endogenous intracellular porphyrin molecules in the bacteria by continuous wave visible light irradiation may result in the production of reactive oxygen species (ROS), predominantly singlet oxygen, and consequently, damage to the DNA and the death of bacteria [30-35]. Therefore, the other mechanism which can contribute to the inactivation of Salmonella typhimurium by a visible USP laser is the photo-production of ROS.

\section{Prospects of the selective disinfection of pathogens by USP lasers}

In the following sections, we discuss a few of the potential applications we envision for this USP laser technology.

\section{Decontamination of blood products for transfusion}

Millions of red blood cell, platelet, plasma and coagulation factor transfusions are performed every year in the United States alone. Implementation of specific donor screening criteria together with nucleic acid and immunologic testing have significantly reduced the risk of transmission of blood components through transfusion for a number of pathogens. This system, however, does not solve all problems posed by pathogens. This is because (1) not all recognized threats have been adequately addressed; (2) there exists a "window period" for a donor during which the infection cannot be detected by testing but during which the donor may be infectious; and (3) screening and tests can only be performed for those pathogens that have been recognized and for which tests are available. Unknown/emerging pathogens will remain as a threat as evidenced by the emergence of HIV and WNV in the past [36]. Therefore, from the transfusion recipient's viewpoint, the ideal strategy for ensuring transfusion safety of blood components should be to implement a preemptive pathogen reduction (PR) technology, which can universally eliminate microbes in a blood product without chemicals and without adversely affecting the function of the blood product itself. For details of all the currently available PR techniques for the disinfection of blood components, please refer to [37-42]. PR technique in plasma components are dominated by solvent detergent treatment [43], methylene blue method [44] and UV-activated photochemical method [45-47] 
such as using amotosalen and riboflavin. Although these are effective in pathogen reduction, some concerns still exist. Several PR treatments have been developed for platelets. Because these treatments share the use of UV light, although at different wavelengths, possible damage to the blood product and/or microbial resistance becomes a concern. Techniques for PR in red blood cells are largely still under development. A significant concern of the above-mentioned techniques is the addition of foreign chemicals which cannot be completely removed after the treatments. These residual chemicals may have short or long term adverse effects on patients who require frequent transfusion of blood components.

In contrast, the chemical-free USP laser technology has been shown to kill $3-5 \log _{10}$ of a variety of pathogens (see Table 1), and more importantly, it exhibits selectivity for microbes over desirable proteins and mammalian cells (see Table 2). Therefore, the USP laser technology represents a plausible pathogen inactivation technology for pathogen reduction of blood products.

\section{Sterilization of biologicals and pharmaceuticals}

Biologicals and pharmaceuticals used in the clinic as well as reagents or cell cultures used in research laboratories can be contaminated with microbes such as Mycoplasma spp., viruses and bacteria, which can affect their safety profile and their biological function. Traditionally, enveloped viruses or bacteria can be killed by the addition of detergent or alcohol-based chemicals. Non-enveloped viruses are harder to kill and are usually inactivated by either heating or using bleach; however, either the heating process or the addition of such chemicals raises the concern of potential side effects. Filtration is an effective way of removing pathogens; however, it is not applicable when the size of undesired pathogen(s) is comparable to that of the desired product. In these cases, a technique that can non-invasively sterilize a solution containing a desired reagent, cell culture, or pharmaceutical without changing the product's structure or function is desirable.

In this regard, USP laser technology represents a plausible method for accomplishing sterilization of biologicals, pharmaceuticals, cell cultures, and reagents. Our preliminary results suggest that a visible USP laser can be used to inactivate viral particles and bacteria, without altering the structure of individual protein units [10]. Therefore, USP laser technology could conceivably be useful for sterilizing biologicals, pharmaceuticals, cell cultures, and reagents.

\section{Generation of efficient and safe vaccines}

The use of killed or attenuated whole microorganisms is an attractive strategy for the development of immunogenic vaccines for many diseases including tuberculosis and malaria [48]. Whole organism vaccines include most of the relevant antigens and retain many of the immunostimulatory components necessary to induce a strong and specific immune response. Various techniques have been applied to this end, including chemical killing, [49] $\mathrm{UV} /$ psoralen treatment [48] and gamma-ray irradiation [50]. Chemical methods such as the application of formalin have the advantages of being simple and cost effective; however, it is not as efficient as other methods. Furthermore, the addition of chemicals raises concerns of potential side effects. UV/psoralen treatment has been shown to be promising in generating killed but metabolically active pathogen vaccines in mouse models; however, the added chemicals are very difficult to remove completely. This raises the concern of potential adverse effects when applied in the clinic. Gamma ray irradiation has been demonstrated to be effective in generating inactivated vaccines in mouse models; however, the gamma-ray photon is high-energy ionizing radiation which will break any chemical bonds in its path including covalent, ionic, and hydrogen bonds in the microorganism. As a result, the use of gamma-ray treated vaccines raises concerns that "new chemical species" may be created that may have adverse effects in humans.

We envision that the use of USP lasers to generate whole inactivated vaccines could be advantageous over current methods, partly because the technique kills the organism efficiently with potentially minimal changes to antigenic and/or immunostimulatory structures, [3-10] and partly because no potentially toxic chemicals are added or created. As a matter of fact, our preliminary results (not shown here) with a USP laser-inactivated H1N1 flu vaccine demonstrates vaccine-induced T-cell responses and protection against challenge in a mouse model.

\section{Potential experimental layout}

One possible approach of using the USP laser technology for selective PR of blood components and pharmaceuticals, and for vaccine production described above is to use a syringe pump to channel the samples through narrow tubing for laser irradiation (see Figure 8).

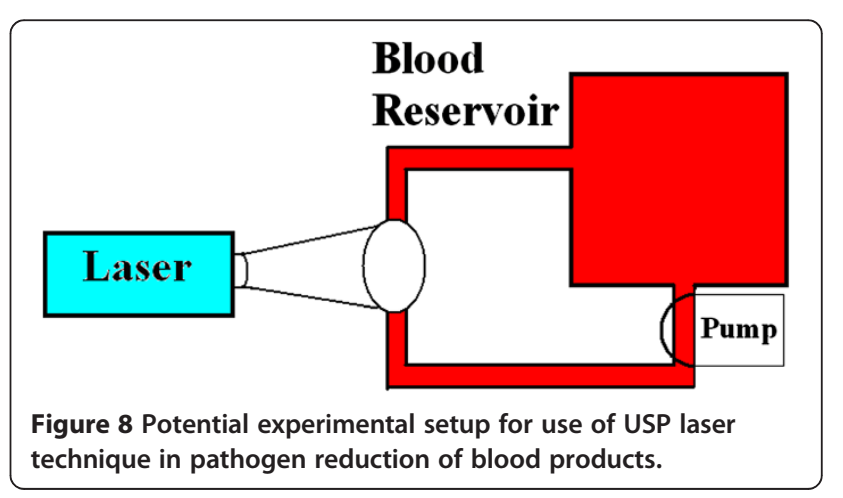




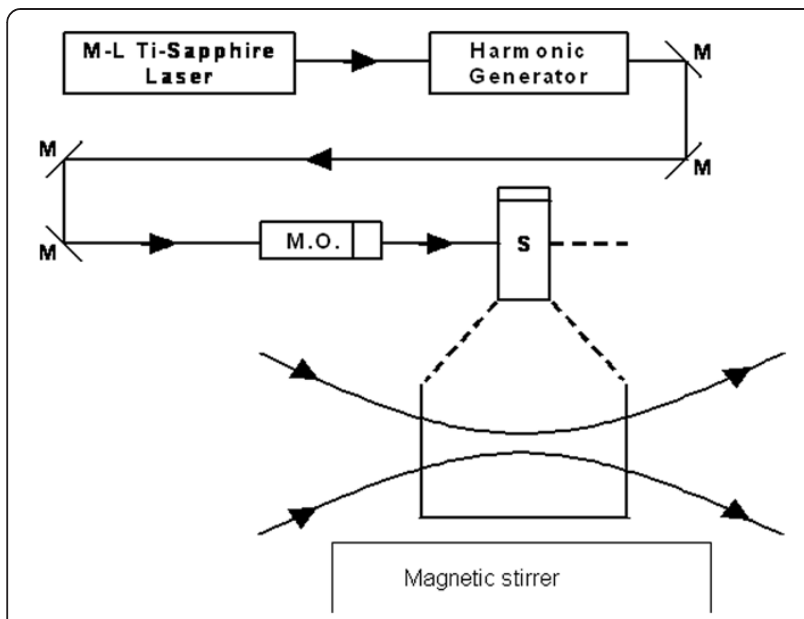

Figure 9 Potential experimental set up for the inactivation of viral particles and bacteria with an USP laser. M.O.: focusing lens; M: mirror; S: vial containing viruses/bacteria in buffer solutions.

If an intense USP laser system is available, an alternative experimental setup involving a magnetic stirrer such as that in Figure 9 can be used.

\section{Conclusion}

The emergence of drug-resistant microbes and new, heretofore-unknown pathogens has renewed the search for effective antimicrobial technologies. The recently developed USP laser technique for microbial load reduction could represent a universal, non-invasive, and environmentally friendly method for selective inactivation of microbes without the use of clinically toxic or environmentally damaging agents. We predict that the USP laser technology will be used for (1) Decontamination of blood products for transfusion; (2) Sterilization of biologicals, pharmaceuticals, cell cultures, and reagents; and (3) Generation of efficient and safe vaccines in the near future.

\section{Competing interests}

The authors declare that they have no competing interests.

\section{Authors' contributions}

SWDT proposed the idea of pathogen inactivation by ultrashort pulsed lasers, performed laser irradiation experiments, carried out the assays and drafted the manuscript. TCW participated in the assays and discussions. JGK participated in the assay and discussions. KTT proposed the idea of pathogen inactivation by ultrashort pulsed lasers, performed laser irradiation experiments, and drafted the manuscript. All authors read and approved the final manuscript.

\section{Acknowledgements}

The authors would like to thank Stuart M. Lindsay, Sara Vaiana, Chien-Fu Hung, Karen Kibler and Bert Jacobs for their contributions to this line of research. The research was funded by the National Science Foundation. The opinions or assertions contained herein are the private views of the authors and are not to be construed as official or reflecting the views of the Armed Forces Radiobiology Research Institute, Uniformed Services University of the Health Sciences, or the U.S. Department of Defense.

\section{Author details}

${ }^{1}$ Department of Radiology, Washington University School of Medicine, St. Louis, MO 63110, USA. ${ }^{2}$ Departments of Pathology, Johns Hopkins School of Medicine, Baltimore, MD 21231, USA. ${ }^{3}$ Departments of Oncology, Johns Hopkins School of Medicine, Baltimore, MD 21231, USA. ${ }^{4}$ Obstetrics and Gynecology, Johns Hopkins School of Medicine, Baltimore, MD 21231, USA. ${ }^{5}$ Molecular Microbiology and Immunology, Johns Hopkins School of Medicine, Baltimore, MD 21231, USA. ${ }^{6}$ Scientific Research Department, Armed Forces Radiobiology Research Institute, 8901 Wisconsin Avenue, Bethesda, MD 20889-5603, USA. 7 Department of Medicine, Uniformed Services University of the Health Sciences, 4301 Jones Bridge Road, Bethesda, MD 20889-5603, USA. ${ }^{8}$ Department of Radiation Biology, Uniformed Services University of the Health Sciences, 4301 Jones Bridge Road, Bethesda, MD 20889-5603, USA. ${ }^{\circ}$ Department of Physics, Arizona State University, Tempe, AZ 85287, USA.

Received: 5 June 2012 Accepted: 13 June 2012 Published: 6 July 2012

\section{References}

1. Weiss RA: How does HIV cause AIDS? Science 1993, 260(5112):1273-1279.

2. Goodnough LT: Risks of blood transfusion. Anesthesiology clinics of North America 2005, 23(2):241-252.

3. Tsen KT, Tsen S-WD, Fu Q, Lindsay SM, Kibler K, Jacobs B, Wu T-C, Karanam B, Jagu S, Roden R, Hung C-F, Sankey O, Ramakrishna B, Kiang JG: Photonic approach to the selective inactivation of viruses with a near-infrared subpicosecond fiber laser. J. Biomedical Optics 2009, 14(7 pages):064042.

4. Tsen KT, Tsen S-WD, Chang C-L, Hung C-F, Wu TC, Kiang JG: Inactivation of viruses by coherent excitations with a low power visible femtosecond Laser. Virology J 2007, 4(1-5):50.

5. Tsen KT, Tsen S-WD, Chang C-L, Hung C-F, Wu TC, Kiang JG: Inactivation of viruses with a very low power visible femtosecond laser. J. Phys: Condensed Matter 2007, 19(1-9):322102.

6. Tsen KT, Tsen S-WD, Sankey OF, Kiang JG: Selective inactivation of microorganisms with near-infrared femtosecond laser pulses. J. Phys: Condensed Matter 2007, 19(1-7):472201.

7. Tsen KT, Tsen S-WD, Chang C-L, Hung C-F, Wu TC, Kiang JG: Inactivation of viruses by laser-driven coherent excitations via impulsive stimulated Raman scattering process. J. Biomedical Optics 2007, 12(1-6):064030.

8. Tsen KT, Tsen S-WD, Chih-Long Chang, Chien-Fu Hung, Wu TC, Ramakrishna B, Mossman K, Kiang JG: In Inactivation of viruses with a femtosecond laser via impulsive stimulated Raman scattering, Proc. of SPIE on Optical Interactions with Tissue and Cells XIX. Vol. 6854, 68540Nth edition. Edited by Jacques SL, Roach WP, Thomas RJ; 2008.

9. Tsen S-WD, Tsen Y-SD, Tsen KT, Wu TC: Selective inactivation of viruses with femtosecond laser pulses and its potential use for in vitro therapy. J. Healthcare Engineering 2010, 1(2):185-196.

10. Tsen KT, Tsen S-WD, Fu Q, Lindsay SM, Zhe Li, Stephanie Cope, Sara Vaiana, Kiang JG: Studies of inactivation of encephalomyocarditis virus, M13 bacteriophage and Salmonella typhimurium by using a visible femtosecond laser irradiation: Insight into the possible inactivation mechanisms. J. Biomedical Optics 2011, 16(1-8):078003.

11. Tsen S-W D, Tsen KT: Inactivation of encephalomyocarditis virus and Salmonella typhimurium by using a visible femtosecond laser. In Proc. of SPIE on Optical Biopsy IX, Vol. 7895, 78950S. Edited by Alfano RR, Demos SG; 2011.

12. Epstein JS, Vostal JG: FDA approach to evaluation of pathogen reduction technology. Transfusion 2003, 43:1347-1349.

13. Yan Y-X, Jr Gamble EB, Nelson KA: Impulsive stimulated scattering: General importance in femtosecond laser pulse interactions with matter, and spectroscopic applications. J Chem Phys 1985, 83:5391-5399.

14. Nelson KA, Miller RJD, Lutz DR, Fayer MD: Optical generation of tunable ultrasonic waves. J Appl Phys 1982, 53:1144-1149.

15. De Silvestri S, Fujimoto JG, Ippen EP, Gamble EB Jr, Williams LR, Nelson KA Femtosecond time-resolved measurements of optic phonon dephasing by impulsive stimulated raman scattering in a-perylene crystal from 20 to $300 \mathrm{~K}$. Chem Phys Lett 1985, 116:146-152.

16. Nelson KA: Stimulated Brillouin scattering and optical excitation of coherent shear Waves. J Appl Phys 1982, 53:6060-6063.

17. Cho GC, Kutt W, Kurz H: Subpicosecond time-resolved coherent-phonon oscillations in GaAs. Phys Rev Lett 1990, 65:764-766. 
18. Cheng TK, Vidal J, Zeiger HJ, Dresselhaus G, Dresselhaus MS, Ippen EP: Mechanism for displacive excitation of coherent phonons in $\mathrm{Sb}, \mathrm{Bi}, \mathrm{Te}$, and Ti2O3. Appl Phys Lett 1991, 59:1923-1925.

19. Chwalek JM, Uher C, Whittaker JF, Mourou GA: Subpicosecond timeresolved studies of coherent phonon oscillations in thin-film YBa2Cu3O6 + x(x<0.4). Appl Phys Lett 1991, 58:980-982.

20. Merlin R: Generating coherent THz phonons with light pulses. Solid State Communications 1997, 102:207-220.

21. Shen YR, Bloembergen N: Theory of simulated Brillouin and Raman scattering. Phys Rev 1965, 137:A1787-A1805.

22. Shen YR: The Principles of Nonlinear Optics. New York: Wiley; 1984.

23. Tsen KT, Tsen S-WD, Dykeman EC, Sankey OF, Kiang JG: In Contemporary Trends in Bacteriophage Research. Edited by Adams Horace T.: Nova Science Publishers, Inc; 2009:151-177. ISBN: 978-1-60692-181-4.

24. Dykeman EC, Sankey OF: Phys. Rev. E 2010, 81:021918.

25. Peeters K, Taormina A, Theor J: Biol. 2009, 256:607-624

26. Janner A: Acta Cryst. 2011, A67:521-532.

27. Tsen KT, Dykeman EC, Sankey OF, Nien-Tsung Lin, Tsen S-WD, Kiang JG: Observation of the low frequency vibrational modes of bateriophage M13 in water by Raman spectroscopy. Virology J 2006, 3(79):1-11.

28. Tsen S-WD, Lin N-T, Kiang JG, Tsen KT, Dykeman EC, Sankey OF: Raman scattering studies of the low frequency vibrational modes of bacteriophage M13 in water - observation of an axial torsion mode. Nanotechnology 2006, 17:5474-5479.

29. Tsen KT, Dykeman EC, Sankey OF: Probing the low frequency vibrational modes of viruses with Raman scattering - bacteriophage M13 in water. J. Biomedical Optics 2007, 12:024009-1-014009-6.

30. Ashkenazi H, Malik Z, Harth Y, Nitzan Y: Eradication of "Propionibacterium acnes by its endogenic porphyrins after illumination with high intensity blue light". FEMS Immunol Med Microbiol 2003, 35:17-24.

31. Elman $M M$, Slatkine $M$, Harth $Y$ : The effective treatment of acne vulgaris by a high-intensity, narrow band 405-420nm light source. J Cosmet Laser Ther 2003, 5:111-116.

32. Feuerstein O, Persman N, Weiss El: Phototoxic effect of visible light on Porphyromonas gingivalis and Fusobacterium nucleatum: an in vitro study. Photochem Photobiol 2004, 80:412-415.

33. Ganz RA, Viveiros J, Ahmad A, Ahmadi A, Khalil A, Tolkoff MJ, Nishioka NS, Hamblin MR: Helicobacter pylori in patients can be killed by visible light. Laser Surg Med 2005, 36:60-265.

34. Soukos NS, Som S, Abernethy AD, Ruggiero K, Dunham J, Lee C, Doukas AG, Goodson JM: Phototargeting oral blackpigmented Bacteria. Antimicrob Agents Chemother 2005, 49:1391-1396.

35. Maclean M, MacGregor SJ, Anderson JG, Woolsey G: High- intensity narrow-spectrum light inactivation and wavelength Sensitivity of Staphylococcus aureus. FEMS Microbiol Lett 2008, 285:227-232.

36. Bryant J, Klein HG, Pathogen Inactivation: The Definitive Safeguard for the Blood Supply. Arch. Pathol. Lab. Med. 2007, 131:719-733.

37. AuBuchon JP: Update on the status of pathogen inactivation methods. ISBT Science Series 2011, 6:181-188.

38. AuBuchon JP: Breathing easy with pathogen inactivation. Blood 2011, 117:749-750.

39. Stramer SL, Hollinger FB, Katz LM, et al: Emerging infectious disease agents and their potential threat to transfusion safety. Transfusion 2009, 49(Suppl. 2):1S-49S.

40. Prowse C: Properties of pathogen-inactivated plasma components. Transf Med Rev 2009, 23:124-133.

41. Pelletier JP, Transue S, Snyder EL: Pathogen inactivation techniques. Best Pract Res Clin Haematol 2006, 19:205-24242.

42. Rock G: A comparison of methods of pathogen inactivation of FFP. Vox Sang 2011, 100:169-178.

43. Horowitz B, Bonomo R, Prince AM, et al: Solvent detergenttreated plasma. A virus-inactivated substitute for fresh frozen plasma. Blood 1992, 79:826-833.

44. Williamson LM, Cardigan R, Prowse PV: Methylene-blue-treated freshfrozen plasma: what is its contribution to blood safety. Transfusion 2003, 43:1322-1329.

45. Larrea L, Calabuig M, Rolda'n V, et al: The influence of riboflavin photochemistry on plasma coagulation factors. Transf Apheresis 2009, 41:199-204
46. Bihm DJ, Ettinger A, Buytaert-Hoefeb KA, et al: Characterization of plasma protein activity in riboflavin and UV light-treated fresh frozen plasma during 2 years of storage at $-30^{\circ} \mathrm{C}$. Vox Sang 2010, 98:108-115.

47. Smith J, Rock G: Protein quality in Mirasol pathogen reduction technology-treated, apheresis-derived fresh-frozen plasma. Transfusion 2010, 50:926-931.

48. Brockstedt DG, Bahjat KS, Giedlin MA, Liu W, Leong M, Luckett W, Gao Y, Schnupf P, Kapadia D, Castro G, Lim JYH, Sampson-Johannes A, Herskovits AA Stassinopoulos A, Archie Bouwer HG, Hearst JE, Portnoy DA, Cook DN, Dubensky TW Jr: Killed but metabolically active microbes: a new vaccine paradigm for eliciting effector T-cell responses and protective immunity. Nature Medicine 2005, 11:853-860.

49. Geeraedts F, Goutagny N, Hornung V, Severa M, de Haan A, Pool J, Wilschut J, Fitzgerald KA, Huckriede A: Superior Immunogenicity of Inactivated Whole Virus H5N1 Influenza Vaccine is Primarily Controlled by Toll-like Receptor Signalling. PLoS Pathogens 2008, 4(8):e1000138.

50. Alsharifi M, Furuya Y, Bowden TR, Lobigs M, Koskinen A, Regner M, Trinidad L, Boyle DB, Mullbacher A: Intranasal Flu Vaccine Protective against Seasonal and H5N1 Avian Influenza Infections. PLoS One 2009, 4(4):e5336.

doi:10.1186/1423-0127-19-62

Cite this article as: Tsen et al:: Prospects for a novel ultrashort pulsed laser technology for pathogen inactivation. Journal of Biomedical Science 2012 19:62.

\section{Submit your next manuscript to BioMed Central and take full advantage of:}

- Convenient online submission

- Thorough peer review

- No space constraints or color figure charges

- Immediate publication on acceptance

- Inclusion in PubMed, CAS, Scopus and Google Scholar

- Research which is freely available for redistribution 